\title{
Effect of early enteral nutrition on laparoscopic common bile duct exploration with enhanced recovery after surgery protocols
}

\author{
Hu Qiang ${ }^{1} \cdot$ Sun Yuanshui ${ }^{1}$
}

Received: 30 October 2019 / Revised: 4 November 2019 / Accepted: 16 January 2020 / Published online: 29 January 2020

(c) Springer Nature Limited 2020

\section{To the Editor:}

With the global promotion of enhanced recovery after surgery (ERAS), Xiang et al. [1] research is a particularly meaningful study. Although we believe it is a very interesting topic, we do not agree on some points and conclusions that are derived from this study, as explained below.

First, the author did not compare the postoperative blood amylase in the two groups. We know that it is easy to induce pancreatitis after endoscopic retrograde cholangiopancreatography (ERCP). For example, in the early enteral nutrition group, patients with potential pancreatitis may be present after ERCP. These people induce pancreatitis after eating early, but not very severe pancreatitis. These people may have abdominal distention after eating early. In the control group, the eating time was later, the pancreas was fully rested, and those with potential pancreatitis recovered due to adequate rest of the pancreas. If this is the case, the probability of abdominal distention in the EEN group is higher than that in the control group, but the pancreatic related factors are not considered in this paper.

Second, the ERAS concept runs through preoperative, intraoperative, and postoperative different treatments at each stage may affect the recovery of postoperative intestinal function. For example, reducing the use of opioids during anesthesia can shorten the postoperative intestinal recovery time and reduce the incidence of abdominal distention. Our previous study [2] also found that early postoperative out of bed activities can accelerate the recovery of intestinal function and reduce the incidence of abdominal distention. However, the article did not compare the time of early postoperative out of bed activities and the use of anesthetic drugs in the two groups.

Third, the author emphasizes that the enteral nutrition formula of the two groups is the same, but does not introduce in detail whether parenteral nutrition is used and whether the calories given by the two groups are the same. In the early stage, the gastrointestinal function of the patient has not fully recovered. It is difficult to meet the energy requirement by using only enteral nutrition. Therefore, extra parenteral nutrition must be given, so the parenteral nutrition formula of the two groups in the article also needs to be specified.

There are many interference factors in this study, so we need to exclude some irrelevant factors, so as to better guide the application of early enteral nutrition in laproscopic common bill due exploration.

\section{Compliance with ethical standards}

Conflict of interest The authors declare that they have no conflict of interest.

Publisher's note Springer Nature remains neutral with regard to jurisdictional claims in published maps and institutional affiliations.

\section{References}

1. Xiang Q, Yuan H, Cai W, Qie S. Effect of early enteral nutrition on laparoscopic common bile duct exploration with enhanced recovery after surgery protocols. Eur J Clin Nutr. 2019;73:1244-9.

2. Hu Q, He T, Sun Y, Wang F, Wu J. Effect of fast-track surgery on inflammatory response and immune function in patients with laparoscopic distal gastrectomy. Eur Surg. 2019;51:254-60.
Sun Yuanshui

15658827827@163.com

1 Department of General Surgery, Tongde Hospital of Zhejiang Province, Zhejiang 310012, China 